\title{
ORT_12 - Osteopontin-a enhances cytoskeleton remodeling and activates intermediate epithelial mesenchymal properties in c643 thryoid cancer cells
}

Etel Rodrigues Pereira Gimba ${ }^{1}$; Ana Emília Goulart Lemos ${ }^{2 *}$; Luciana Bueno Ferreira ${ }^{3}$; Raquel Lima ${ }^{4}$; Catarina Tavares ${ }^{4}$; Rui Batista ${ }^{4}$; Paula Soares ${ }^{4}$.

${ }^{1}$ UFF/Pólo Universitário de Rio das Ostras;

${ }^{2} \mathrm{UFF}$ - Universidade Federal Fluminense;

${ }^{3} \mathrm{INCa}$;

${ }^{4}$ IPATIMUP, Universidade do Porto, Portugal.

Introduction: Thyroid cancer (TC) is the most common endocrine malignancy and the majority of these tumors derive from follicular cells, and can vary from differentiated to undifferentiated cell phenotypes. Several gene products are aberrantly expressed in TC, including osteopontin (OPN). Total OPN is a key activator of epithelial-mesenchymal transition (EMT) and stemness potential, modulating cancer progression and related features. OPN primary transcript undergoes alternative splicing, generating OPN splice variants (OPN-SV), mainly OPNa, OPNb and OPNc, which are aberrantly expressed in cancer cells, presenting tumor and tissue specific roles. Our group previously demonstrated that OPNa overexpression in c643 TC cells can activate cell migration and invasion.However, the cellular and molecular mechanisms related to these events and how OPNa isoform can differently contribute to these aspects is still very poorly understood.

Objective: This work aimed to investigate the morphological cell features and the related transcriptional and/or protein level profiling of cytoskeleton, EMT and stemness markers that could be associated to OPNa-induced migratory properties in c643 TC cells.

Methodology: c643 cell line overexpressing OPNa (c643-OPNa) and the control c643 cells containing the empty vector (c643-EV) were used to analyze the expression of cytoskeletal, EMT and stemness markers by quantitative real time PCR (RT-qPCR), immunoblot and/or immunofluorescence. Thyrosphere formation assays were also performed using both cell clones, besides the evaluation of stemness transcriptional patterns by RT-qPCR.

Results: c643-OPNa displayed increased cell spreading and activated focal adhesion formation, besides an enlarged vimentin intermediate filament network distribution, when compared to c643-EV. These cell features were similarly stimulated by conditioned media (CM) secreted by c643-OPNa. In addition, at the transcriptional level, c643-OPNa cells expressed both epithelial and mesenchymal markers, with $\mathrm{N}$-cadherin and Twist being upregulated in relation to c643-EV. Moreover, among 4 tested stemness markers, SOX-2 and LIN-28 were upregulated. Furthermore, c643-OPNa showed increased protein expression levels of N-cadherin, besides upregulation of the stemness markers c-Myc, SOX-2, NANOG and OCT-4A, when compared to c643-EV. The c643-OPNa also renderedhigher number and size of formed thyrospheres, which also expressed higher levels of stemness markers.

Conclusion: Our data evidenced that OPNa overexpression can enhance cell spreading and focal adhesion formation, besides vimentin network remodeling, which are features compatible with cells with higher migration rates. Moreover, according to observed transcriptional and protein expression patterns of EMT and stemness markers, OPNa overexpression in c643 cells may be associated with an intermediate EMT phenotype and higher stemness features. Altogether, these morphological and molecular features could be among the signals differentially activated by OPNa-overexpressing cells that may evoke theacquisition of TC cell motility and invasion profile. These assumptions may support the proposal of OPNa as a putative target to therapeutic approaches aiming to inhibit TC motility and invasion properties.

Keywords: Thyroid cancer; Osteopontin-a; Epithelial Mesenchymal Transition 